\title{
Tanshinone IIA arrests cell cycle and induces apoptosis in 786-O human renal cell carcinoma cells
}

\author{
XIANLI WEI ${ }^{1,2}$, LUNBIN ZHOU ${ }^{2}$, LIANGYONG HU ${ }^{2}$ and YAOXIONG HUANG ${ }^{1}$ \\ ${ }^{1}$ Department of Biomedical Engineering, Jinan University, Guangzhou, Guangdong 510632; \\ ${ }^{2}$ Guangzhou Institute of Measuring and Testing Technology, Guangzhou, Guangdong 510030, P.R. China
}

Received November 26, 2011; Accepted January 30, 2012

DOI: $10.3892 / \mathrm{ol} .2012 .626$

\begin{abstract}
Tanshinone IIA (Tan IIA), isolated from the Chinese medicinal herb Danshen, has been reported to have anticancer effects in several tumor models, while its effects on renal cell carcinoma have not been studied. In the present study, we evaluated the effects of Tan IIA on growth inhibition and apoptosis in the renal cancer cell line 786-O and its mechanism of action. Results of the MTT assay indicated that the treatment of 786-O cells with Tan IIA resulted in a concentration-dependent decrease in cell viability. Flow cytometry analysis revealed that Tan IIA treatment caused apoptosis following cell cycle perturbation. Furthermore, we examined the expression of cell cycle and apoptosis-related proteins using immunoblotting, which indicated an upregulation of p53, p21, bax and caspase-3 in Tan IIA-treated cells compared with Tan IIA-untreated cells. These results suggest that the activation of p53 and the upregulation of its target genes, including p21 and bax, may be involved in the mitochondrial apoptosis induced by Tan IIA in 786-O cells.
\end{abstract}

\section{Introduction}

Renal cell cancer (RCC) is a cause of mortality worldwide and its incidence rate continues to rise. Although several therapeutic approaches have been applied to the treatment of RCC, including surgery, immunological therapies and vaccine treatment (1), further improvements are needed. Notably, the modulation of the apoptotic response provides new prospects for therapeutic strategies in cancer $(2,3)$. Thus, novel and promising anticancer drugs may be identified by further investigations into the induction of apoptosis.

Tanshinone IIA (Tan IIA, 14,16-epoxy-20-nor$5(10), 6,8,13,15$-abietapentaene-11,12-dione), one of the phytochemical compounds isolated from the Chinese medicinal herb Danshen (root of Salvia miltiorrhiza Bunge),

Correspondence to: Dr Yaoxiong Huang, Department of Biomedical Engineering, Jinan University, Guangzhou, Guangdong 510632, P.R. China

E-mail: yaoxionghuang@163.com

Key words: tanshinone IIA, renal cell carcinoma, apoptosis, p53 possesses anti-inflammatory (4,5) and antioxidant properties $(6,7)$. Tan IIA has also been shown to have anticancer activity through the induction of apoptosis in a variety of cancers (8-10). However, it remains unclear whether Tan IIA is capable of inhibiting cell growth and inducing apoptosis in human RCC cells. In the present study, we investigated the effect of Tan IIA on human RCC 786-O cells in vitro and the mechanisms by which it functions.

\section{Materials and methods}

Reagents. Tan IIA (>99\% pure) and MTT were purchased from Sigma Chemical Co. (St. Louis, MO, USA). The annexin V-FITC apoptosis detection kit was purchased from BD Biosciences (San Jose, CA, USA). The antibodies used in this study were: monoclonal anti-p53 (sc-126, Santa Cruz Biotechnology, Inc., Santa Cruz, CA, USA), polyclonal antip21 (71-1000, Zymed Laboratories, San Francisco, CA, USA), polyclonal anti-bax (2772, Cell Signaling Technology, Inc., MA, USA), polyclonal anti-caspase-3 (Upstate Biotechnology, Lake Placid,NY,USA) and monoclonal anti-actin (ms-1295-po, NeoMarkers, Fremont, CA, USA).

Cell culture. The human RCC cell line 786-O was provided by Dr Jun Hu (Sun Yat-sen University, Guangzhou, China) and cultured in Dulbecco's modified Eagle's medium (DMEM) supplemented with $10 \%$ fetal bovine serum (FBS) and $100 \mathrm{U} / \mathrm{ml}$ penicillin and streptomycin.

MTT assay. 786-O cells were seeded in 96-well microtitre plates with $1 \times 10^{3}$ cells/well and incubated for $24 \mathrm{~h}$ in $100 \mu \mathrm{l}$ culture medium. The cells in the experimental group were then treated with 1,2, 4 or $8 \mu \mathrm{g} / \mathrm{ml}$ of Tan II-A for $24 \mathrm{~h}$. MTT [100 $\mu \mathrm{l}$ $(5 \mathrm{~g} / \mathrm{l})$ ] was added to the cells which were then cultivated for a further $4 \mathrm{~h}$. Following the removal of the supernatant fluid, $100 \mu \mathrm{l} /$ well DMSO was added to the cells which were agitated for $15 \mathrm{~min}$. The absorbance was measured at $570 \mathrm{~nm}$ by an ELISA reader. The untreated 786-O cells served as controls. Each assay was repeated three times.

Immunoblotting (IB). The cell lysates were boiled with 3X SDS loading buffer and then fractionated by SDS-PAGE. The proteins were transferred to a PVDF membrane, which was then incubated with a primary specific antibody in $5 \%$ 


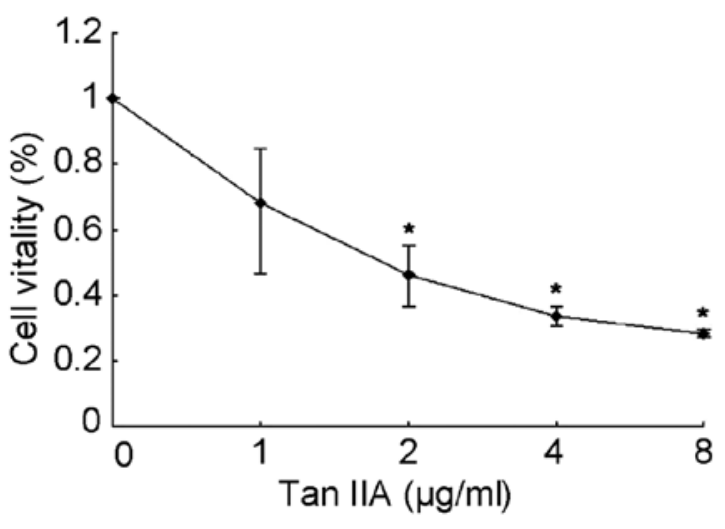

Figure 1. Cytotoxicity effects of Tan IIA on 786-O cells. 786-O cells were treated with Tan IIA $(0,1,2,4$ and $8 \mu \mathrm{g} / \mathrm{ml})$ for $24 \mathrm{~h}$. Cell viability was measured by MTT assay. The data are presented as the mean $\pm \mathrm{SD} .{ }^{*} \mathrm{P}<0.05$. Tan IIA, tanshinone IIA.

milk, followed by horse radish peroxidase (HRP)-conjugated anti-mouse or anti-rabbit antibodies and ECL detection reagent (Amersham Life Science, Piscataway, NJ, USA).

Cell cycle distribution analysis. Cells were plated in 6-well culture dishes at concentrations determined to yield 60-70\% confluence within $24 \mathrm{~h}$. The cells were then treated with Tan IIA $(0,2,4$ or $8 \mu \mathrm{g} / \mathrm{ml})$. After $24 \mathrm{~h}$, the cells were washed twice with PBS and then centrifuged. The pellet was fixed with $70 \%$ ethanol at $4^{\circ} \mathrm{C}$. The ethanol was washed away and the cells were treated with $40 \mathrm{mg} / \mathrm{ml}$ propidium iodide (PI) and $0.1 \mathrm{mg} / \mathrm{ml} \mathrm{RNase}$ (Boehringer, Germany) for $30 \mathrm{~min}$ at $37^{\circ} \mathrm{C}$. The cells $\left(2 \times 10^{4}\right)$ were analyzed and the DNA content was measured using a FACStar cytofluorometer (BD Biosciences) equipped with an argon-ion laser at $488 \mathrm{~nm}$.

Apoptosis assessed by flow cytometry. The extent of apoptosis was evaluated by annexin V-FITC and flow cytometry. The cells were grown at a density of $1 \times 10^{6}$ cells in 6-well culture dishes and were treated with Tan IIA $(0,2,4$ or $8 \mu \mathrm{g} / \mathrm{ml})$ for $24 \mathrm{~h}$. Following treatment, the cells were harvested, washed twice with pre-chilled PBS and resuspended in $1 \mathrm{X}$ binding buffer at a concentration of $1 \times 10^{6}$ cells $/ \mathrm{ml}$. This solution (100 $\mu \mathrm{l}$ ) was mixed with $5 \mu \mathrm{l}$ annexin V-FITC and $5 \mu \mathrm{l}$ PI for $15 \mathrm{~min}$, then $400 \mu \mathrm{l} 1 \mathrm{X}$ binding buffer was added. The analysis was carried out using a FACStar cytofluorometer with CXP software.

Statistical analysis. Values are presented as the mean \pm SD of the control. The Student's t-test was used to analyze the statistical significance between the Tan IIA-treated and control groups. $\mathrm{P}<0.05$ was considered to indicate a statistically significant result.

\section{Results}

The effects of Tan IIA on the viability of 786-O cells. To examine the cytotoxicity of Tan IIA on the human RCC cell line 786-O, 786-O cells were cultured and treated with Tan IIA. The cytotoxicity of Tan IIA in 786-O cells was detected using the MTT assay. The percentages of viable cells relative to the control (DMSO)-treated cells were 68.2, 46.4, 33.4 and $28.3 \%$ when cultured with Tan IIA $(1,2,4$ and $8 \mu \mathrm{g} / \mathrm{ml}$, respectively) for $24 \mathrm{~h}$. The half maximal inhibitory concentration $\left(\mathrm{IC}_{50}\right)$ value for Tan IIA treatment for $24 \mathrm{~h}$ was estimated to be $2 \mu \mathrm{g} /$ $\mathrm{ml}$. The results show that Tan IIA treatment induced a marked dose-dependent inhibition of the growth of 786-O cells (Fig. 1).

Tan IIA induces 786- $O$ cell cycle arrest in the $S$ phase. To determine the effect of Tan IIA on the growth of 786-O cells, the cell cycle distribution was analyzed by flow cytometry. When $786-\mathrm{O}$ cells were treated with Tan IIA $(0,2,4$ and $8 \mu \mathrm{g} / \mathrm{ml})$ for $24 \mathrm{~h}$, the percentage of cells in the $\mathrm{S}$ phase was $10.0,11.5$, 20.4 and $23.3 \%$, respectively (Fig. 2). The results indicate the inhibition of the growth of 786-O cells following treatment with Tan IIA.

Tan IIA-induced apoptosis of 786-O cells. Since cell cycle perturbations may mediate the induction of apoptosis, we examined the effect of Tan IIA on the apoptosis of 786-O cells using the annexin V/PI approach. It was observed that, following the treatment of 786-O cells for $24 \mathrm{~h}$ with Tan IIA ( 0 , $2,4$ and $8 \mu \mathrm{g} / \mathrm{ml})$, the number of early apoptotic cells increased to $8.6,27.2,27.8$ and $28.8 \%$, respectively. The number of late apoptotic cells increased to 2.9, 9.2, 12.5 and $13.9 \%$, respectively. The total percentage of apoptotic cells was directly related to the Tan IIA concentration, increasing from $11.5 \%$ (control) to $36.4,40.3$ and $42.7 \%(2,4$ and $8 \mu \mathrm{g} / \mathrm{ml}$ Tan IIA, respectively; Fig. 3), consistent with the results of the cytotoxicity assay. The results revealed that Tan IIA induced cell apoptosis of 786-O cells in a concentration-dependent manner.

Gene expression profile correlated with growth inhibition and apoptosis following Tan IIA treatment. Since Tan IIA treatment caused $\mathrm{S}$ phase cell cycle arrest, we examined the effect of Tan IIA on cell cycle regulatory molecules which are active in the $S$ phase. IB indicated that Tan IIA treatment induced the upregulation of the $\mathrm{p} 21$ protein, a key regulator of $\mathrm{S}$ phase cell cycle transition (11), in a dose-dependent manner. In order to establish the involvement of a mitochondrial apoptotic event, the level of the bax and caspase-3 proteins was measured. As shown in Fig. 4, Tan IIA treatment resulted in a significant dose-dependent increase in the level of bax and caspase-3.

p53, an upstream regulator of p21 and bax (12), is involved in cell cycle arrest and apoptotic induction in response to cell stress. In the present study, we also assessed the level of p53 expression by IB following Tan IIA treatment. The data indicate that the level of the p53 protein increased markedly following treatment with Tan IIA.

\section{Discussion}

Tan IIA, traditionally administered to treat cardiovascular disease (13), has gained much attention for its anticancer properties in cell culture and animal carcinogenesis models (8-10). Although previous studies have shown the chemochemical/ therapeutic potential of Tan IIA against several cancer types, information regarding the apoptotic effects of Tan IIA in human RCC cells is unavailable. The present study provides evidence for the first time that Tan IIA has an anticancer effect on human RCC. 

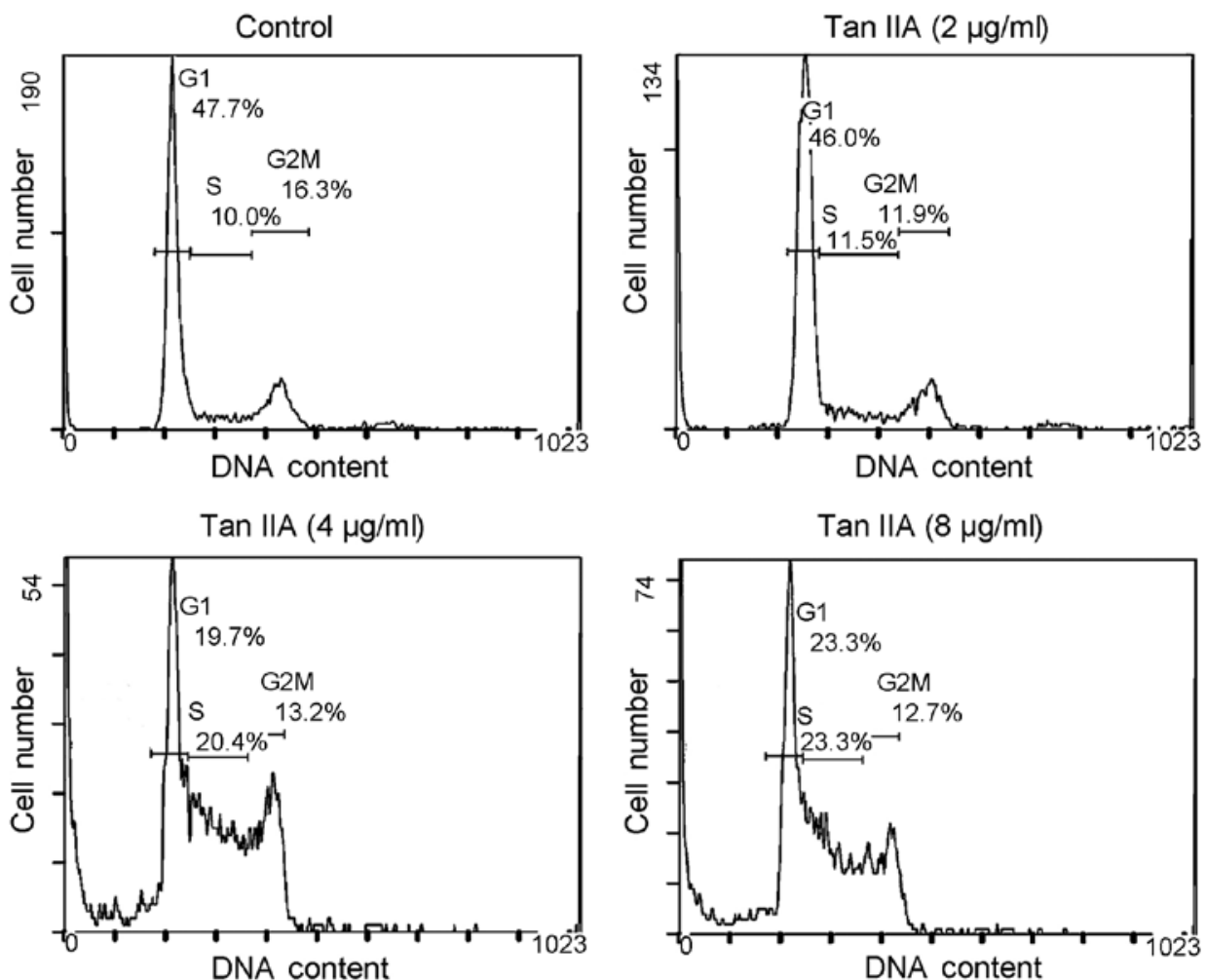

Figure 2. Cell cycle analysis of 786-O cells treated with Tan IIA. Cells were cultured with different concentrations of Tan IIA for 24 h and then stained with propidium iodide. The DNA content was analyzed by flow cytometry. The S phase arrest was enhanced with increasing concentrations of Tan IIA. Tan IIA, tanshinone IIA.
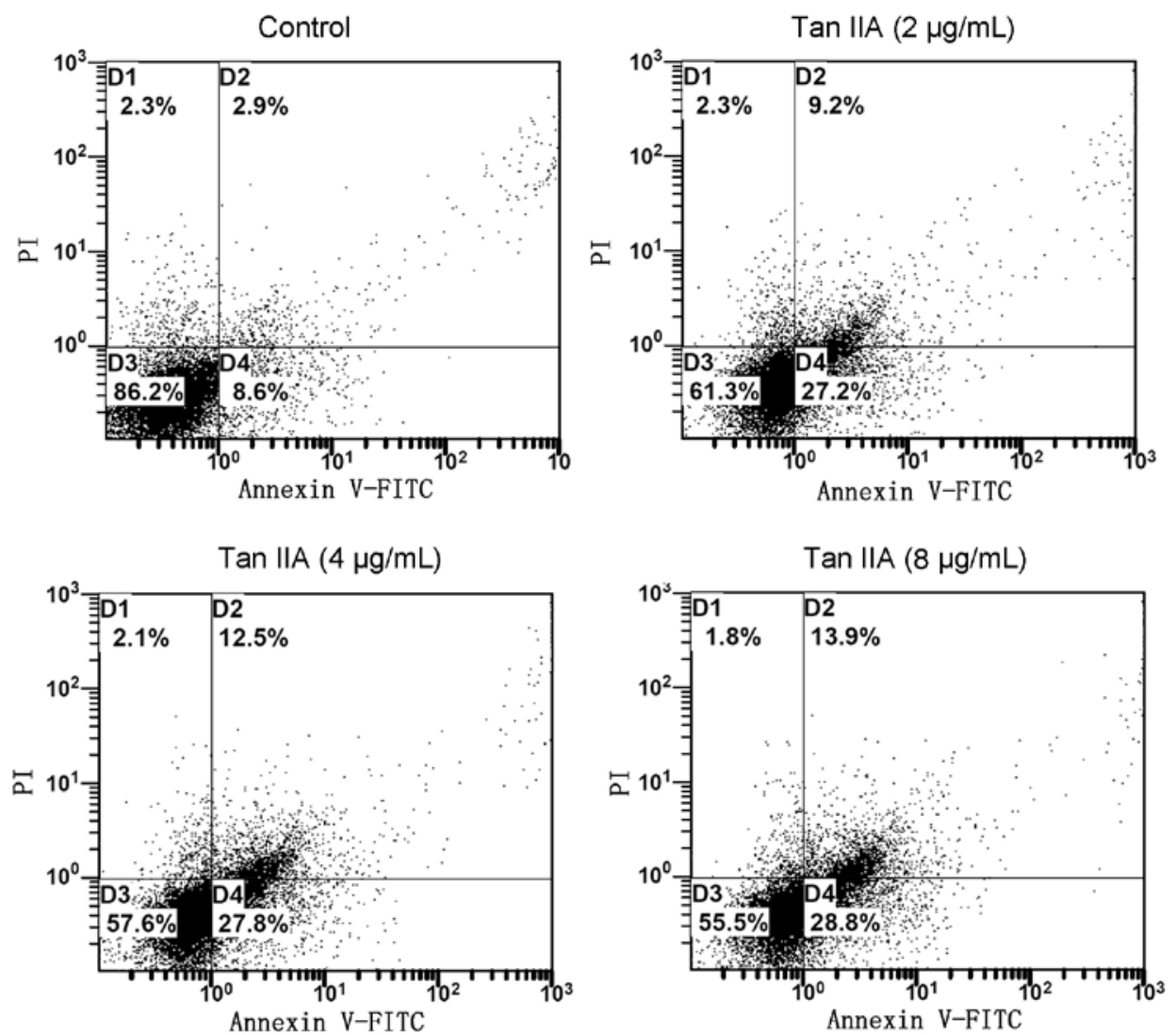

Figure 3. Dose-dependent apoptosis induced by treatment with Tan IIA in 786-O cells. Cells treated with various concentrations of Tan IIA were double-stained with annexin V and PI and analyzed by flow cytometry. The gate setting distinguished between living (bottom left), necrotic (top left), early apoptotic (bottom right), and late apoptotic (top right) cells. Tan IIA, tanshinone IIA; PI, propidium iodide. 




Figure 4. Expression of the proteins changed in 786-O cells treated with different concentrations of Tan IIA for $24 \mathrm{~h}$. Tan IIA treatment resulted in the upregulation of p53 and its target genes, p21 and bax. Subsequently, caspase- 3 was increased with the induction of apoptosis by Tan IIA. Tan IIA, tanshinone IIA.

A number of studies have demonstrated that Tan IIA is able to reduce cell vitality due to its cytotoxic properties (14). The results of the MTT assay in our study also showed that treatment with Tan IIA resulted in a marked and dose-dependent inhibition of the growth of 786-O cells. We observed a slight enhancement of vitality of 786-O cells following trace Tan IIA $(<0.05 \mu \mathrm{g} / \mathrm{ml})$ treatment compared with untreated cells. Tan IIA is a well-established antioxidant present in the Chinese medicinal herb Danshen (root of Salvia miltiorrhiza Bunge) (15). Therefore, trace amounts of Tan IIA may promote cell growth. By contrast, treatment with a high dose of Tan IIA generally exerts cytotoxicity, resulting in a reduction of cell vitality. Furthermore, the analysis of the cell cycle distribution revealed that Tan IIA treatment induced $\mathrm{S}$ phase arrest in 786-O cells. As p21, a cyclin-dependent kinase (CDK) inhibitor, is crucial in retarding S phase (11), we analysed its expression and found that it was dose-dependently upregulated following Tan IIA treatment. Moreover, Tan IIA treatment led to a concentration-dependent increase in the levels of $\mathrm{p} 53$, paralleling the accumulation of $\mathrm{p} 21$. These results suggest that the observed p 21 accumulation may be due to p53 activation, since p53 (wild-type) upregulates p21 expression (16). It should be noted that it is possible that the level of p21 was altered via a p53-independent pathway.

In addition to promoting cell cycle arrest, $\mathrm{p} 53$ targets genes with apoptotic activity, among them the bax gene (12). The expression level of bax was assessed using IB. The results revealed that bax was upregulated in parallel with p53 activation in Tan IIA-induced apoptosis in RCC cells, suggesting that the mitochondrial apoptotic pathway is involved in Tan IIA-induced cell death. The mitochondrial apoptotic process eventually leads to the activation of the caspases, which are both initiators and effectors of the apoptotic programmed cell death. Similar to the observations of bax, treatment with Tan IIA significantly increased the expression level of caspase-3 in 786-O cells (Fig. 4). Notably, p53 targets the

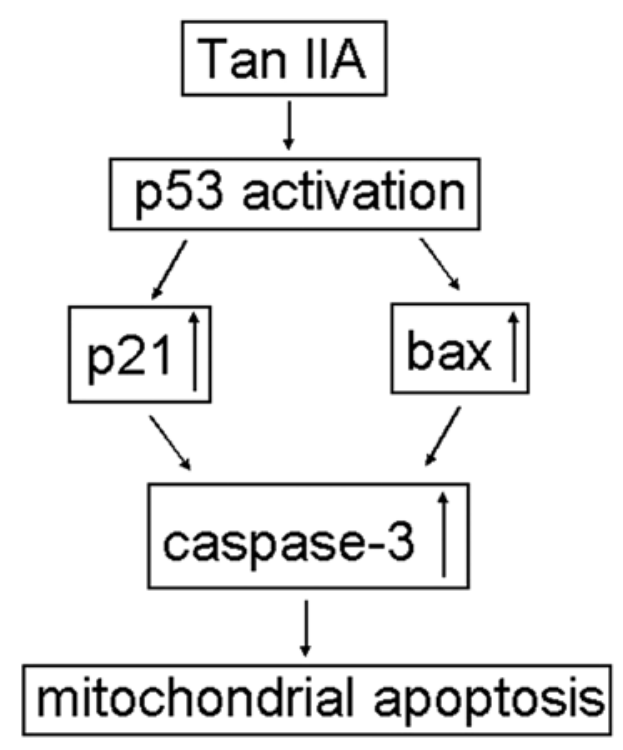

Figure 5. Schematic model for the mechanism of Tan IIA-induced apoptosis in 786-O cells. Tan IIA, tanshinone IIA.

Fas gene, which is involved in the mitochondria-independent pathway of apoptosis (17). In our study, we did not detect any change in the level of expression of Fas, indicating that the specific cell death signals used by Tan IIA appear to be dependent on the cell type.

In conclusion, we report that human RCC 786-O cells in culture respond to Tan IIA treatment by showing a reduction in growth and an increase in apoptotic cell death. Furthermore, IB results revealed that Tan IIA activates p53 expression and subsequently induces the upregulation of $\mathrm{p} 21$ and bax. These findings suggest that Tan IIA induced apoptosis in 786-O cells, possibly through both the p21-mediated cell cycle perturbation and the mitochondrial-mediated intrinsic cell-death pathways (Fig. 5). These findings suggest the efficacy of Tan IIA as a chemopreventive candidate to exert antigrowth and proapoptotic actions on human RCC.

\section{Acknowledgements}

We thank Dr Jun Hu (Sun Yat-sen University, Guangzhou, China) for providing the human RCC cell line 786-O cells. This study was supported by the National Natural Science Foundation of China (no. 30772492).

\section{References}

1. Bird J and Hayter M: A review of the literature on the impact of renal cancer therapy on quality of life. J Clin Nurs 18: 2783-2800, 2009.

2. Kyprianou N, Martikainen P, Davis L, English HF and Isaacs JT: Programmed cell death as a new target for prostatic cancer therapy. Cancer Surv 11: 265-277, 1991.

3. Kyprianou N, Bains AK and Jacobs SC: Induction of apoptosis in androgen-independent human prostate cancer cells undergoing thymineless death. Prostate 25: 66-75, 1994

4. Jang SI, Kim HJ, Kim YJ, Jeong SI and You YO: Tanshinone IIA inhibits LPS-induced NF-kappaB activation in RAW 264.7 cells: possible involvement of the NIK-IKK, ERK1/2, p38 and JNK pathways. Eur J Pharmacol 542: 1-7, 2006. 
5. Li W, Li J, Ashok M, et al: A cardiovascular drug rescues mice from lethal sepsis by selectively attenuating a late-acting proinflammatory mediator, high mobility group box 1. J Immunol 178: 3856-3864, 2007.

6. Lin R, Wang WR, Liu JT, Yang GD and Han CJ: Protective effect of tanshinone IIA on human umbilical vein endothelial cell injured by hydrogen peroxide and its mechanism. J Ethnopharmacol 108: 217-222, 2006.

7. Wang AM, Sha SH, Lesniak W and Schacht J: Tanshinone (Salviae miltiorrhizae extract) preparations attenuate aminoglycoside-induced free radical formation in vitro and ototoxicity in vivo. Antimicrob Agents Chemother 47: 1836-1841, 2003.

8. Wang X, Wei Y, Yuan S, et al: Potential anticancer activity of tanshinone IIA against human breast cancer. Int J Cancer 116: 799-807, 2005

9. Wang J, Wang X, Jiang S, et al: Growth inhibition and induction of apoptosis and differentiation of tanshinone IIA in human glioma cells. J Neurooncol 82: 11-21, 2007.

10. Chiu TL and Su CC: Tanshinone IIA induces apoptosis in human lung cancer A549 cells through the induction of reactive oxygen species and decreasing the mitochondrial membrane potential. Int J Mol Med 25: 231-236, 2010.
11. Ogryzko VV, Wong P and Howard BH: WAF1 retards S-phase progression primarily by inhibition of cyclin-dependent kinases. Mol Cell Biol 17: 4877-4882, 1997.

12. el-Deiry WS: Regulation of p53 downstream genes. Semin Cancer Biol 8: 345-357, 1998.

13. Sun J, Tan BK, Huang SH, Whiteman M and Zhu YZ: Effects of natural products on ischemic heart diseases and cardiovascular system. Acta Pharmacol Sin 23: 1142-1151, 2002.

14. Lu Q, Zhang P, Zhang X and Chen J: Experimental study of the anti-cancer mechanism of tanshinone IIA against human breast cancer. Int J Mol Med 24: 773-780, 2009.

15. Cao EH, Liu XQ, Wang JJ and Xu NF: Effect of natural antioxidant tanshinone II-A on DNA damage by lipid peroxidation in liver cells. Free Radic Biol Med 20: 801-806, 1996.

16. Yu C, Friday BB, Lai JP, et al: Cytotoxic synergy between the multikinase inhibitor sorafenib and the proteasome inhibitor bortezomib in vitro: induction of apoptosis through Akt and c-Jun NH2-terminal kinase pathways. Mol Cancer Ther 5: 2378-2387, 2006.

17. Müller M, Wilder S, Bannasch D, et al: p53 activates the CD95 (APO-1/Fas) gene in response to DNA damage by anticancer drugs. J Exp Med 188: 2033-2045, 1998. 